\title{
Contourlet Transform for Texture Representation of Ultrasound Thyroid Images
}

\author{
Stamos Katsigiannis, Eystratios G. Keramidas, and Dimitris Maroulis \\ University of Athens, Department of Informatics and Telecommunications, \\ Panepistimioupoli, Ilisia, 15784, Athens, Greece \\ \{stamos, e.keramidas, dmarou\} @di.uoa.gr
}

\begin{abstract}
Texture representation of ultrasound (US) images is currently considered a major issue in medical image analysis. This paper investigates the texture representation of thyroid tissue via features based on the Contourlet Transform (CT) using different types of filter banks. A variety of statistical texture features based on CT coefficients, have been considered through a selection schema. The Sequential Float Feature Selection (SFFS) algorithm with a $k$-NN classifier has been applied in order to investigate the most representative set of $\mathrm{CT}$ features. For the experimental evaluation a set of normal and nodular ultrasound thyroid textures have been utilized. The maximum classification accuracy was $93 \%$, showing that CT based texture features can be successfully applied for the representation of different types of texture in US thyroid images.
\end{abstract}

Keywords: contourlet transform, ultrasound images, feature extraction, thyroid, feature selection.

\section{Introduction}

Modern medical ultrasonography (US) presents a unique set of advantages including real-time data acquisition, low cost, absence of any side effects and high resolution imaging. Thus, US has become the most common imaging modality for certain types of medical examinations, including that of the thyroid gland. Currently a challenge for the image analysis community is the application of automated or semi-automated computational methods on US images, for the computer aided diagnosis (CAD) of different types of diseases. A key issue for any CAD approach remains the texture representation method utilized.

Texture representation is a fundamental issue in image analysis and computer vision. It has been extensively investigated in the literature for more than three decades. Numerous approaches have been proposed dealing with textural features extraction which can be divided into four main categories [1] statistical, signal processing, model-based and geometrical. Signal processing approaches have drawn much attention, resulting in the proposal of a variety of texture representation methods, including the power spectral methods using the Fourier spectrum, the Discrete Cosine Transform (DCT), the Discrete Hartley Transform (DHT) [2], and 
more recently, the Gabor filters [3], the Haar [4] basis functions, the Discrete Wavelet Transform (DWT) and the Contourlet Transform (CT) [5][6].

Although the Discrete Wavelet Transform has been successfully applied for a wide range of image analysis problems, for two dimensions it tends to ignore the smoothness along contours [5]. In addition, the DWT provides only limited directional information which is an important aspect of multidimensional signals [6]. These limitations have been partially addressed by the CT which can efficiently approximate a smooth contour at multiple resolutions. Additionally in the frequency domain, the CT offers a multiscale and directional decomposition, providing anisotropy and directionality, features missing from the DWT [5][6][7]. The CT has been successfully used in a variety of texture analysis applications, including SAR and natural image classification [8], content-based image retrieval [9], image denoising [10], despeckling of images [11], image compression [12], etc.

The aim of this study is to investigate the performance of the Contourlet Transform (CT) for the representation of medical ultrasound (US) textures of the thyroid gland. A set of statistical features calculated from the CT coefficients are evaluated through a supervised classification schema on real thyroid US images. Additionally, a feature selection phase has been applied through the Sequential Float Feature Selection (SFFS) algorithm, for the extraction of the most representative set of CT features.

The rest of this paper is organized in three sections. Section 2 presents the proposed methodology including the $\mathrm{CT}$ and the above mentioned feature extraction schema. This methodology is evaluated through an experimental study on real thyroid ultrasound images presented in section 3, whereas conclusions are presented in section 4.

\section{Methodology}

\subsection{The Contourlet Transform}

The CT is a directional multiresolution image representation scheme proposed by Do and Vetterli [5] which is effective in representing smooth contours in different directions of an image [6], thus providing directionality and anisotropy [5][9]. The method utilizes a double filter bank, in order to obtain a sparse expansion of typical images containing smooth contours. In this filter bank, first the Laplacian Pyramid (LP) is used to detect the point discontinuities of the image and then a Directional Filter Bank (DFB) to link point discontinuities into linear structures. This scheme results in an image expansion that uses basic elements like contour segments and thus it is named CT. The separation of directional and multiscale decomposition stages provides a fast and flexible transform with computational complexity $O(N)$ for $N$ pixel images when using Finite Impulse Response (FIR) filters [6][8].

The LP, introduced in [13], provides a way to obtain multiscale decomposition. In each decomposition level, it creates a downsampled lowpass version of the original image and a bandpass image. A coarse image with the lower frequencies and a more detailed image with the supplementary high frequencies containing the point discontinuities are obtained. This scheme can be iterated continuously in the lowpass image, restricted only from the size of the original image. 
The next step consists of the DFB which is a 2-D directional filter bank proposed by Bamberger and Smith, that can be maximally decimated while achieving perfect reconstruction [6][14]. The original DFB is efficiently implemented via an $l$-level binary tree leading to $2^{l}$ subbands with wedge-shaped frequency partitioning. However, in the CT, a new method is applied [6] which avoids modulating the input image and uses a simpler rule for expanding the decomposition tree [15]. The simplified DFB consists of two stages. First, a two-channel quincunx filter bank [16] that divides the 2-D spectrum into vertical and horizontal directions, and then a shearing operator that just reorders the samples.

Bandpass images from the LP decomposition are fed into a DFB in order to obtain the directional information. This scheme can be iterated on the coarse image and the combined result is a double iterated filter bank, named contourlet filter bank [8], which decomposes images into directional subbands at multiple scales.

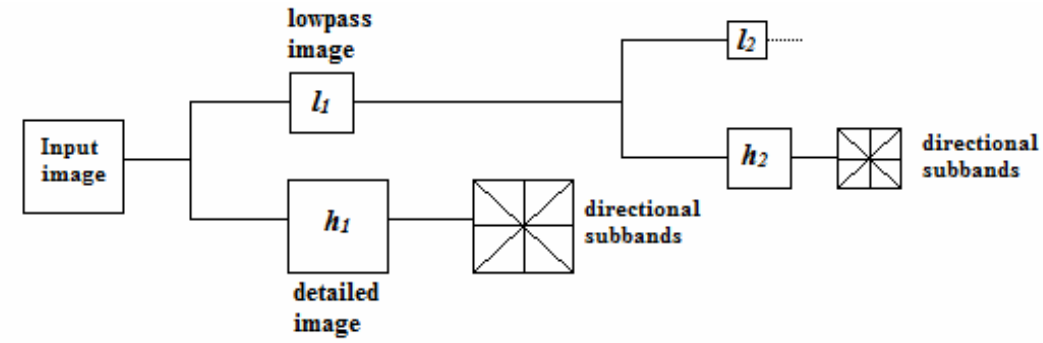

Fig. 1. The contourlet filter bank. Images $l_{i}$ and $h_{i}$ are the lowpass and detailed image, respectively, obtained from the $i$ th level of decomposition with the LP. In every level of LP decomposition, $h_{i}$ is fed into the DFB.

\subsection{Feature Extraction}

The set of statistical texture features in the contourlet domain evaluated in this study, have been proposed by Liu [8]. This set consists of the first order statistical measures that follow:

i) Energy. The energy $E_{j k}$ of a subband image $I_{j k}$ from the CT decomposition is defined as

$$
E_{j k}=\frac{1}{M_{j k} \cdot N_{j k}} \sum_{n=1}^{N_{j k}} \sum_{m=1}^{M_{j k}}\left[I_{j k}(m, n)\right]^{2}
$$

Where $I_{j k}$ is the subband image of the $k$ th direction in the $j$ th level. $M_{j k}$ is the row size and $N_{j k}$ the column size of the subband image $I_{j k}$.

ii) Standard deviation. Provides the means to capture the scale of the diversity of the image. The standard deviation $S_{j k}$ of the subband image $I_{j k}$ is defined as 


$$
\begin{gathered}
S_{j k}=\sqrt{\frac{1}{M_{j k} \cdot N_{j k}} \sum_{n=1}^{N_{j k}} \sum_{m=1}^{M_{j k}}\left(I_{j k}(m, n)-\bar{I}_{j k}\right)^{2}} . \\
\bar{I}_{j k}=\frac{1}{M_{j k} \cdot N_{j k}} \sum_{n=1}^{N_{j k}} \sum_{m=1}^{M_{j k}} I_{j k}(m, n) .
\end{gathered}
$$

iii) Information entropy. For texture images, it represents the complexity of the texture information. The information entropy $H_{j k}$ of a subband image $I_{j k}$ is defined as

$$
\begin{gathered}
H_{j k}=-\sum_{n=1}^{N_{j k}} \sum_{m=1}^{M_{j k}} p_{j k}(m, n) \cdot \log p_{j k}(m, n) . \\
p_{j k}(m, n)=\frac{\left|I_{j k}(m, n)\right|^{2}}{\sqrt{\sum_{n=1}^{N_{j k}} \sum_{m=1}^{M_{j k}}\left[I_{j k}(m, n)\right]^{2}}} .
\end{gathered}
$$

Using the above features, the feature vector for the subband image of the $k$ th direction in the $j$ th level is defined as $f_{j k}=\left(E_{j k}, S_{j k}, H_{j k}\right)$. If the CT is defined as $J$ level and for the $j$ th $(j=1, \ldots, J)$ level a $K_{j}$ bands DFB is applied, then a total number o $K$ subband images is obtained, where

$$
K=\sum_{j=1}^{J} K_{j}
$$

By combining and rearranging all the feature vectors of the $K$ subband images, the feature vector $F=\left\{f_{i}\right\}, i=1,2, \ldots, K$ of the input image is obtained and normalized as proposed in [9].

\subsection{Feature Selection}

Feature selection offers more than one significant advantages, including reduction of computational complexity, improved generalization ability and robustness against outliers. A widely adopted algorithm for feature selection is the sequential floating forward selection (SFFS) algorithm [17] which has been used in a broad range of applications [18]. The idea behind the SFFS algorithm consists of consecutive forward selection steps, followed by a number of backward steps as long as the resulting subsets are better than the previously evaluated ones at the same level. Starting from an initially empty set of features, at each forward step an additional feature for which the classification accuracy is maximized is selected. Respectively, at each backward step the maximum subset that results in an improved classification accuracy is being selected. 


\section{Experimental Evaluation}

Supervised classification experiments have been carried out to evaluate the performance of the proposed texture representation approach. The image set consists of real medical ultrasound images of the thyroid gland. A total of 72 thyroid ultrasound images were obtained from examinations performed on 43 patients, using a Philips HDI 5000 sonographic imaging system, with a spatial resolution of $470 \times 470$ pixels and amplitude resolution of 8 bits. During these examinations the parameters of the sonograph were kept the same. This set of ultrasound images includes hypogenic thyroid nodules, as it has been diagnosed by expert physicians. From each ultrasound image, an equal number of healthy and nodular sample blocks have been selected. The total number of non overlapping 32x32 pixel blocks (Fig. 2) resulting from this process was 200 .

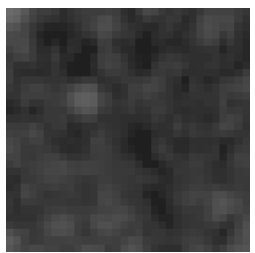

(a)

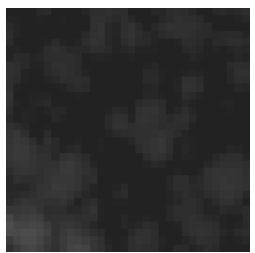

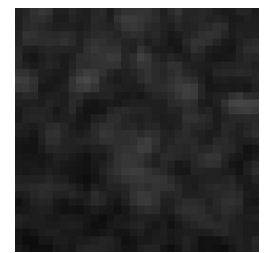

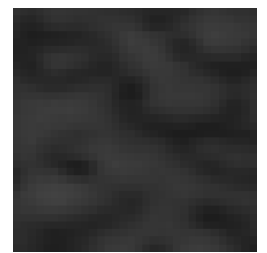

(b)

Fig. 2. Sample images from experimental dataset. (a) Normal and (b) nodular thyroid tissue.

The classification task was implemented by means of the non parametric and generally effective $k$-NN classification approach [24]. The distance measure used by the $k$-NN was the Euclidean and parameter $k$ was $\{3,5\}$. For all experiments conducted, the classification accuracy for all experiments was estimated by 10 -fold cross validation [19].

Due to the small size of thyroid nodules, the sample blocks selected are also small (32x32 pixels) and they did not allow decomposition of more than three levels with the LP and six levels with the DFB. The first LP decomposition level supports up to six levels of DFB decomposition, decreased by one for every extra LP decomposition level. The filters applied for the LP were the filters Burt, 5-3 and 9-7 and for the DFB the 5-3 and 9-7 filters. All possible combinations of filters and decomposition levels were tested.

Detailed results about the maximum classification accuracy obtained using the $k$ NN classifier and different filter combinations are shown in Fig. 3. From this figure it can be noticed that the maximum classification accuracy is $77 \%$. This resulted from the filter 9-7 for the LP and the DFB, with two levels of LP decomposition, decomposed into sixteen and two directional subbands respectively, from finer to coarser scale.

The application of the SFFS selection algorithm led to improved classification results for every experimental setup as shown in Fig. 4. In this case the maximum classification accuracy was $93 \%$ using only 36 out of 205 features and the confusion matrix is presented in Table 1. This accuracy has been obtained via 5-3 filters for the 


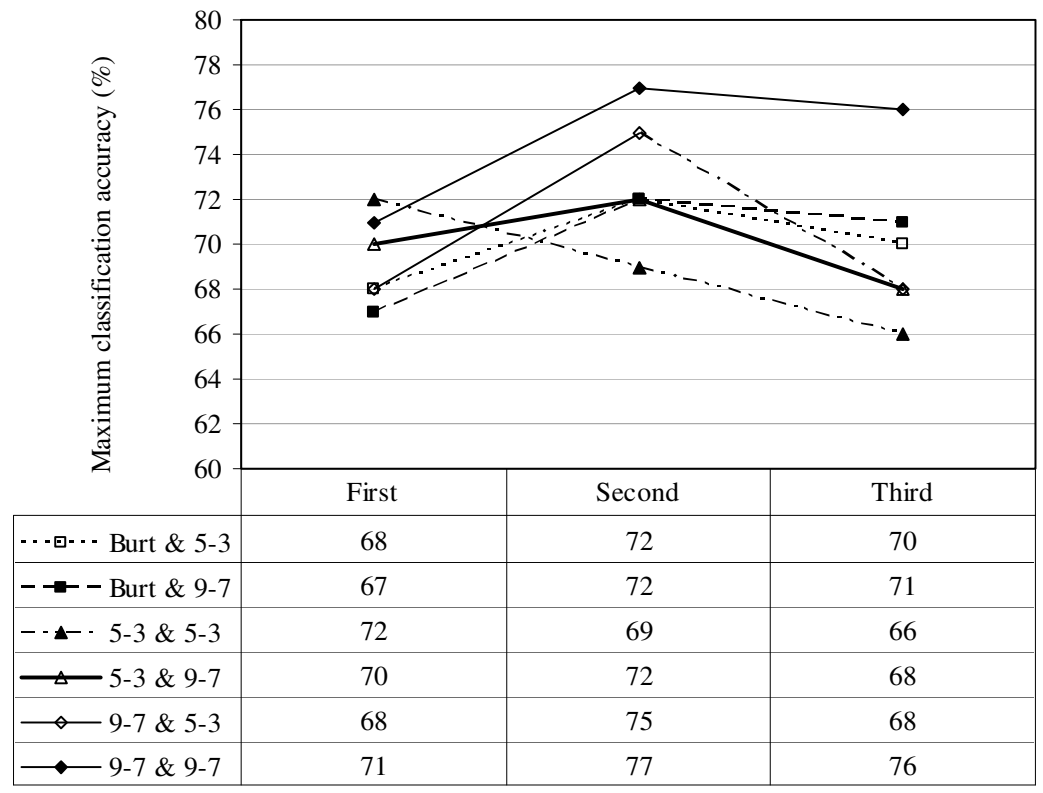

Fig. 3. Maximum classification accuracy of the $k$-NN classifier for each filter combination at each level of LP decomposition (first, second, third). The first filter of the filter combinations refers to the one used for the LP and the second refers to the one used for the DFB.

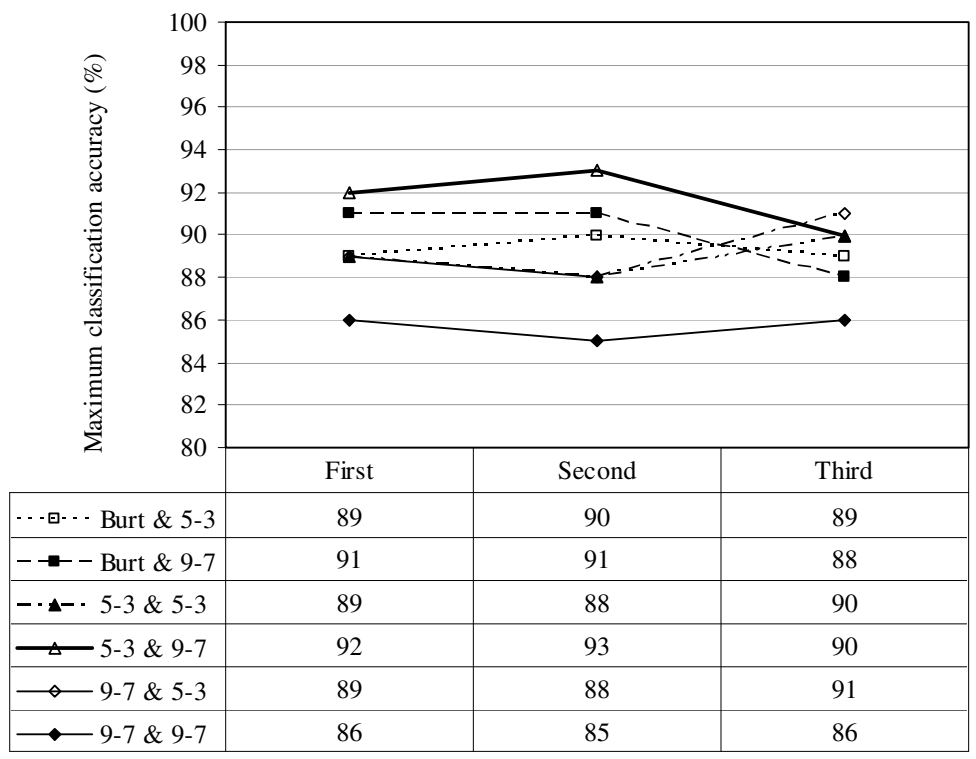

Fig. 4. Maximum classification accuracy of the SFFS algorithm with the $k$-NN classifier, for each filter combination at each level of LP decomposition (first, second, third). The first filter of the filter combinations refers to the one used for the LP and the second refers to the one used for the DFB. 
Table 1. Confusion matrix for the setup which gave the best results (93\% accuracy)

\begin{tabular}{|c|c|c|}
\hline Correct & Nodular & Healthy \\
\hline Nodiction & & $4.4 \%$ \\
\hline Healthy & $2.1 \%$ & $47.9 \%$ \\
\hline
\end{tabular}

LP and the 9-7 filters for the DFB, with two levels of LP decomposition, and then decomposed into sixty four and four directional subbands respectively from finer to coarser scale.

This result shows that a significantly smaller subset of CT features is necessary and sufficient to describe effectively the thyroid US texture.

\section{Conclusions}

In this study a methodology for the texture representation of thyroid tissue in US images has been investigated, utilizing features based on the Contourlet Transform (CT) and various types of filter banks. The experimental evaluation through supervised classification on real US thyroid images led to promising results. Furthermore, through a feature selection phase the maximum classification accuracy reached $93 \%$ for a significantly smaller set of features. These results are considered to provide evidence for the effectiveness of CT texture representation of US thyroid images. As overall conclusion, it can be argued that the combinations of 5-3 and 9-7 filters resulted in better classification accuracy for the second level of LP decomposition. Future work could include US images of higher resolution and the evaluation of different types of statistical features.

\section{Acknowledgement}

This work was partially supported by the National and Kapodistrian University of Athens Special Account of Research Grants. We would also like to thank EUROMEDICA S.A. Greece for the provision of part of the medical images.

\section{References}

1. Tuceryan, M., Jain, A.K.: Texture Analysis. In: The Handbook of Pattern Recognition and Computer Vision, 2nd edn., pp. 207-248. World Scientific Publishing Co., Singapore (1998)

2. Manian, V., Vasquez, R., Katiyar, P.: Texture Classification Using Logical Operators. IEEE Transactions on image processing 9(10) (October 2000)

3. Pichler, O., Teuner, A., Hosticka, B.J.: A comparison of texture feature extraction using adaptive Gabor filtering, pyramidal and tree structured wavelet transforms. Pattern Recognition 29, 733-742 (1996)

4. Lonnestad, T.: A new set of texture features based on the Haar transform. In: 11th Int. Conf. Acoustics, Speech, Signal Processing, vol. 4, pp. 661-664 (1992) 
5. Do, M.N., Vetterli, M.: Contourlets: A Directional Multiresolution Image Representation. In: Proc. of IEEE International Conference on Image Processing (ICIP), Rochester (2002)

6. Do, M.N., Vetterli, M.: The contourlet transform: an efficient directional multiresolution image representation. IEEE Trans. Im. on Proc. 14(12), 2091-2106 (2005)

7. Po, D.D.-Y., Do, M.: Directional multiscale modelling of images using the contourlet transform. IEEE Transactions on Image Processing 15(6), 1610-1620 (2006)

8. Liu, Z.: Minimum Distance Texture Classification of SAR Images in Contourlet Domain. In: 2008 International Conference on Computer Science and Software Engineering (2008)

9. Srinivasa rao, C., Srinivas kumar, S., Chatterji, B.N.: Content Based Image Retrieval using Contourlet Transform. ICGST-GVIP Journal 7(3) (2007)

10. Tsakanikas, P., Manolakos, E.S.: Improving 2-DE gel image denoising using contourlets. Proteomics 9(15), 3877-3888 (2009)

11. Varshney, L.R.: Despeckling Synthetic Aperture Radar Imagery using the Contourlet Transform. Application of Signal Processing (April 2004)

12. Karras, D.A., Karkanis, S.A., Mertzios, B.G.: Image Compression Using the Wavelet Transform on Textural Regions of Interest. In: $24^{\text {th }}$ Euromicro Conf., vol. 2, pp. 633-639 (1998)

13. Burt, P.J., Adelson, E.H.: The Laplacian Pyramid as a Compact Image Code. IEEE Trans. on Communications, 532-540 (1983)

14. Bamberger, R.H., Smith, M.J.T.: A filter bank for the directional decomposition of images: Theory and design. IEEE Trans. Signal Proc. 40(4), 882-893 (1992)

15. Shapiro, J.M.: Embedded image coding using zerotrees of wavelet coefficients. IEEE Trans. on Sign. Proc., Wavelets and Signal Processing 41(12), 3445-3462 (1993)

16. Vetterli, M.: Multidimensional subband coding: Some theory and algorithms. Signal Proc. 6(2), 97-112 (1984)

17. Pudil, P., Novovicova, J., Blaha, S.: Statistical approach to pattern recognition: Theory and practical solution by means of PREDITAS system. Kyber. 27(1), 78 (1991)

18. Ververidis, D., Kotropoulos, C.: Fast Sequential Floating Forward Selection applied to emotional speech features estimated on DES and SUSAS data collections. In: Proc. European Signal Processing Conf. (EUSIPCO), Italy (2006)

19. Theodoridis, S., Koutroumbas, K.: Pattern Recognition, 3rd edn. Academic Press, London (2006) 